\title{
Bayesian inference to study genetic control of resistance to gray leaf spot in maize
}

\author{
M. Balestre ${ }^{1}$, R.G. Von Pinho ${ }^{2}$, A.H. Brito ${ }^{1}$ \\ ${ }^{1}$ Departamento de Biologia, Universidade Federal de Lavras, \\ Lavras, MG, Brasil \\ ${ }^{2}$ Departamento de Agricultura, Universidade Federal de Lavras, \\ Lavras, MG, Brasil \\ Corresponding author: M. Balestre \\ E-mail: marciobalestre@hotmail.com
}

Genet. Mol. Res. 11 (1): 17-29 (2012)

Received June 27, 2011

Accepted September 23, 2011

Published January 9, 2012

DOI http://dx.doi.org/10.4238/2012.January.9.3

\begin{abstract}
Gray leaf spot (GLS) is a major maize disease in Brazil that significantly affects grain production. We used Bayesian inference to investigate the nature and magnitude of gene effects related to GLS resistance by evaluation of contrasting lines and segregating populations. The experiment was arranged in a randomized block design with three replications and the mean values were analyzed using a Bayesian shrinkage approach. Additive-dominant and epistatic effects and their variances were adjusted in an over-parametrized model. Bayesian shrinkage analysis showed to be an excellent approach to handle complex models in the study of genetic control in GLS, since this approach allows to handle overparametrized models (main and epistatic effects) without using model-selection methods. Genetic control of GLS resistance was predominantly additive, with insignificant influence of dominance and epistasis effects.
\end{abstract}

Key words: Cercospora zeae-maydis; Genetic resistance; Zea mays; Bayesian inference 


\section{INTRODUCTION}

Gray leaf spot (GLS, Cercospora zeae-maydis) is currently one of the major foliar maize diseases in Brazil due to its nation-wide distribution and level of damage in susceptible hybrids (Brito et al., 2008). As of 2000, GLS has reached epidemic proportions in several regions in Brazil (Julliati et al., 2004).

In Brazil, there is consensus among maize breeders that a major cause of interruptions in the planting of commercial maize hybrids is the severity of diseases such as GLS. The emergence of variations in the pathogen population was mainly due to the cultivation of susceptible hybrids and to changes in production systems. The need for the development of hybrids with genetic resistance to this disease is evident. Fortunately, the efficiency of this strategy was confirmed in the U.S. and Africa, where damages caused by this disease were reported ahead of its occurrence in Brazil (Menkir and Ayodele, 2005; Derera et al., 2008). However, it is important to note that the germplasm used in most reports in the literature about the genetic control of GLS was adapted to ecological conditions different from those in Brazil. Thus, there is little information about the study of inheritance of GLS resistance in maize using contrasting lines and its hybrid generations.

The studies on the type of gene action involved in GLS resistance indicate the predominance of additive gene action (Menkir and Ayodele, 2005; Derera et al., 2008). However, some reports have stated significance of non-additive effects, i.e., to fully explain the mode of inheritance of GLS resistance, dominance effects and epistatic effects should be included in a genetic-statistical modeling (Coates and White, 1998).

The effort of any breeding program may be facilitated if information on the genetic control of the trait of interest is available. A powerful tool to study the genetic inheritance of traits is the use of phenotypic data from segregating populations tracing back to pure and contrasting parental lines, enabling breeders to choose the most appropriate selection strategy.

To study the inheritance of any trait, join-scaling tests have usually been applied where main and epistatic effects can be adjusted in the single-model and tested by the chi-square test (Mather and Jinks, 1971). This methodology has some limitations, mainly when the degrees of freedom are restricted to number of parameters adjusted in the full-model, i.e., epistatic effects. One alternative to get around this limitation is to perform model selection or adjusting complex models where the number of parameters is higher than the number of observations. Taking into account the latter one, $\mathrm{Xu}$ (2003) proposed an approach to deal with complex models in the quantitative trait locus (QTL) analysis where the number of parameters is higher than the number of observations. In this approach, each parameter is assumed as random variables with specific variance, if so, those parameters with small variance will have its effect shrunk close to zero.

The $\mathrm{Xu}$ (2003) approach is a free-model selection methodology and was applied in this study. In addition, some authors have proposed the use of Bayesian inference to study the effect of major genes or polygenes in the genetic control of several traits (Janss et al., 1997; Kadarmideen and Janss, 2005). In this circumstance, Xu (2003) methodology could be very attractive since it enables to adjust additive, dominant, epistatic effects and their variances in a single-model.

Regarding the GLS resistance, no study, to date, has been developed using Bayesian inference. In this context, the present study was carried out to investigate the nature and magnitude of gene effects related to resistance of GLS based on Bayesian inference by the evaluation of contrasting lines and its segregating populations. 


\section{MATERIAL AND METHODS}

Four populations $\left(\mathrm{F}_{1}, \mathrm{~F}_{2}, \mathrm{BC}_{11}\right.$, and $\mathrm{BC}_{21}$ ) tracing back to two backgrounds (GNS30 $\mathrm{x}$ GNS31 and GNS84 x GNS31) and its parental inbred lines (GNS30, GNS31 and GNS84) were evaluated. The inbred lines GNS30 and GNS31 came from the same background formed by lines derived from the Cateto and Caribbean genotypes. Both have hard grains, short stature and a medium-late cycle. GNS31 is susceptible to GLS and GNS30 is GLS-resistant. The GNS84 line was obtained from the selfing of varieties derived from genotype Tuxpeno with semi-dent grain, medium sized, early maturity and GLS resistance. The seeds of the parent lines, as well as the $\mathrm{F}_{1}, \mathrm{~F}_{2}, \mathrm{BC}_{11}$, and $\mathrm{BC}_{21}$ generations, were obtained in the 2007/2008 growing season.

The experiments were sown in November and December 2008, with a 30-day interval between the first and second sowing. The experimental area is part of the Federal University of Lavras, MG (lat. $21^{\circ} 14$ 'S; long. $45^{\circ} 00^{\prime} \mathrm{W} ; 918 \mathrm{~m}$ asl). The local climate is Cwa (subtropical with rainy summer and dry winter). Two experiments were conducted separately for each sowing date, one for each background in a randomized complete block design with three replications. Each replication consisted of 11 plots; one for each parental lines and the $\mathrm{F}_{1}$ generation, two for $\mathrm{BC}_{11}$, two for $\mathrm{BC}_{21}$, and four for the $\mathrm{F}_{2}$ generation. Thus, it was always possible to use plots of equal size, with four $5-\mathrm{m}$ long rows, spaced $0.8 \mathrm{~m}$ apart. This resulted in a greater representation of segregating populations, with around 80 plants per plot.

On both sowing dates, $400 \mathrm{~kg}$ /ha fertilizer formulation $8-28-16+0.5 \%$ zinc was applied to both experiments. When the plants had 4-5 leaves, $300 \mathrm{~kg} / \mathrm{ha}$ NPK fertilizer 30-00-20 was applied as side-dressing. The second top-dressing of $100 \mathrm{~kg} / \mathrm{ha}$ urea was applied when the plants had 8-9 leaves. Cultural treatments as well as pest control were applied according to crop requirements.

The disease process was initiated by natural infection. To assess disease severity, 20 plants of each plot were labeled for data collection during flowering. The evaluation was performed 95 days after plant emergence, as indicated by Brito et al. (2008), who found this season to be more efficient to discriminate the resistance level of genotypes. For these evaluations of disease, severity data (grades) were represented by the percentage of infected leaf area (ILA) on a 1-9 rating scale (Von Pinho et al., 2001) as follows: $1=0 \%$ ILA and no symptoms; $2=\leq 1 \%$ ILA with a few scattered lesions; $3=1-20 \%$ ILA; $4=20-40 \%$ ILA; $5=40-50 \%$ ILA with lesions on the ear leaf and a few lesions on leaves above the ear; $6=50-60 \%$ ILA with lesions on the leaves above the ear; $7=60-75 \%$ ILA; $8=75-90 \%$ ILA, and $9=\geq 90 \%$ ILA with premature plant death prior to physiological maturity (formation of black layer on grain).

The mean data of each generation, within two seasons and considering the two bakground, were obtained. This approach presented two consequences, i.e., the accuracy of the means data was different and the matrix of genetic effects was assumed as known (fixed). Thus, one can assume that the phenotypic means are given by:

$$
y \mid b, a, d, a a, a d, d d, \sigma^{2} V \sim N\left(b+Z_{1} a+Z_{2} d+Z_{3} a a+Z_{4} a d+Z_{5} d d, \sigma_{e}^{2} V\right) \quad \text { (Equation 1) }
$$

where $y$ is the vector of population means within each background; $b$ is the vector of general mean; $a$ is the additive effect; $d$ is the dominance effect, and $a a, a d$ and $d d$ are the additive $\mathrm{x}$ additive, additive $\mathrm{x}$ dominant and dominant $\mathrm{x}$ dominant epistatic effects, respectively; $Z$ are vectors taken as known and reflect the expectation of the effects $(a, d, a a, a d, d d)$ on the populations $\mathrm{P}_{1}, \mathrm{P}_{2}, \mathrm{~F}_{1}, \mathrm{~F}_{2}, \mathrm{RC}_{1}$, and $\mathrm{RC}_{2} ; \sigma^{2}$ is the residual variance, and $V$ the matrix of weights 
attributed to each mean. These weights take into account the phenotypic variance associated with each value on each generation and mean number of sampled individuals per generation, background and season. The diagonal matrix of weights $\mathrm{V}\left(1 / \mathrm{w}_{\mathrm{ii}}\right)$ was constructed so that the trace of $\mathrm{V}^{-1}$ corresponded to the sample size.

In Bayesian shrinkage analysis suggested by Xu (2003) for QTL analysis each variable is taken as random realization with specific variance. Thus, for general mean, it was assumed a non-informative prior distribution given by:

$$
\begin{gathered}
p(a) \propto N\left(0, \sigma_{a}^{2}\right), p(d) \propto N\left(0, \sigma_{d}^{2}\right), p(a a) \propto N\left(0, \sigma_{a a}^{2}\right), p(a d) \propto N\left(0, \sigma_{a d}^{2}\right), p(d d) \propto N\left(0, \sigma_{d d}^{2}\right), \\
p\left(\sigma_{a}^{2}\right) \propto 1 / \sigma_{a}^{2}, p\left(\sigma_{d}^{2}\right) \propto 1 / \sigma_{d}^{2}, p\left(\sigma_{a a}^{2}\right) \propto 1 / \sigma_{a a}^{2}, p\left(\sigma_{a d}^{2}\right) \propto 1 / \sigma_{a d}^{2}, p\left(\sigma_{d d}^{2}\right) \propto 1 / \sigma_{d d}^{2} \text { and } \\
p\left(\sigma^{2}\right) \propto 1 / \sigma^{2} .
\end{gathered}
$$

Once all distributions are taken as known, the Monte Carlo Markov Chain method (MCMC) via Gibbs sampling can be implemented. Thus, the conditional posterior for the general mean was assumed as normal with mean and variance given by:

$$
\begin{array}{cc}
b=(1 / n) \sum_{i=1}^{n} V_{i i}^{-1}\left(y_{i}-Z_{1 i} a-Z_{2 i} d-Z_{3 i} a a-Z_{4 i} a d-Z_{5 i} d d\right) & \text { (Equation 2) } \\
\text { and }(1 / n) \sigma^{2} & \text { (Equation 3) }
\end{array}
$$

The conditional posteriors for additive and dominant effects are normal with mean and variance given by:

$$
\begin{array}{cc}
a=\left(Z_{1} V^{-1} Z_{1}+\sigma^{2} / \sigma_{a}^{2}\right)^{-1} Z_{1} V^{-1}\left(y-b-Z_{2} d-Z_{3} a a-Z_{4} a d-Z_{5} d d\right) & \text { (Equation 4) } \\
d=\left(Z_{2} V^{-1} Z_{2}+\sigma^{2} / \sigma_{d}^{2}\right)^{-1} Z_{2} V^{-1}\left(y-b-Z_{1} a-Z_{3} a a-Z_{4} a d-Z_{5} d d\right) & \text { (Equation 5) } \\
\text { and variances }\left(Z_{1} V^{-1} Z_{1}+\sigma^{2} / \sigma_{a}^{2}\right)^{-1} \sigma^{2} & \text { (Equation 6) } \\
\text { and }\left(Z_{2} V^{-1} Z_{2}+\sigma^{2} / \sigma_{d}^{2}\right)^{-1} \sigma^{2}, \text { respectively. } & \text { (Equation 7) }
\end{array}
$$

The conditional posteriors for the additive $\mathrm{x}$ additive, additive $\mathrm{x}$ dominant and dominant $\mathrm{x}$ dominant epistatic effects and their variances are given by:

$$
\begin{array}{cr}
a a=\left(Z_{3} V^{-1} Z_{3}+\sigma^{2} / \sigma_{a a}^{2}\right)^{-1} Z_{3} V^{-1}\left(y-b-Z_{1} a-Z_{2} d-Z_{4} a d-Z_{5} d d\right) & \text { (Equation 8) } \\
a d=\left(Z_{4} V^{-1} Z_{4}+\sigma^{2} / \sigma_{a d}^{2}\right)^{-1} Z_{4} V^{-1}\left(y-b-Z_{1} a-Z_{2} d-Z_{3} a a-Z_{5} d d\right) & \text { (Equation 9) } \\
d d=\left(Z_{5} V^{-1} Z_{5}+\sigma^{2} / \sigma_{d d}^{2}\right)^{-1} Z_{5} V^{-1}\left(y-b-Z_{1} a-Z_{2} d-Z_{3} a a-Z_{4} a d\right) & \text { (Equation 10) }
\end{array}
$$

and variances

$$
\left(Z_{3} V^{-1} Z_{3}+\sigma^{2} / \sigma_{a a}^{2}\right)^{-1} \sigma^{2}
$$

(Equation 11) 


$$
\begin{gathered}
\left(Z_{4} V^{-1} Z_{4}+\sigma^{2} / \sigma_{a d}^{2}\right)^{-1} \sigma^{2} \\
\left(Z_{5} V^{-1} Z_{5}+\sigma^{2} / \sigma_{d d}^{2}\right)^{-1} \sigma^{2}
\end{gathered}
$$

The residual, additive, dominant, additive $\mathrm{x}$ additive, additive $\mathrm{x}$ dominant, and dominant $\mathrm{x}$ dominant variances were samples from inverted chi-square distributions, that is:

$$
\begin{aligned}
\sigma_{a}^{2} & =a^{2} / \chi_{1}^{2} \\
\sigma_{d}^{2} & =d^{2} / \chi_{1}^{2} \\
\sigma_{a a}^{2} & =a a^{2} / \chi_{1}^{2} \\
\sigma_{a d}^{2} & =a d^{2} / \chi_{1}^{2} \\
\sigma_{d d}^{2} & =d d^{2} / \chi_{1}^{2}
\end{aligned}
$$

(Equation 18)

where $\chi_{1}^{2}$ is sampled from the chi-square distribution with one degree of freedom and

$$
\sigma^{2}=\sum_{i=1}^{n}\left(y_{i}-b-Z_{i 1} a-Z_{i 2} d-Z_{i 3} a a-Z_{i 4} a d-Z_{i 5} d d,\right)^{2} / \chi_{n}^{2}
$$

where $\chi_{n}^{2}$ is sampled from the chi-square distribution with $n$ degree of freedom being $n$ the number of genetic populations under analysis. In this study $n=6$. The MCMC process consists in sampling the general mean, additive, dominant, and epistatic effects from Equations 2 to 13 and additive, dominant, epistatic, and residual variances from Equations 14 and 19.

This methodology can readily be applied to single analysis, i.e., one sown season and one background. For join analysis - two sown and two backgrounds - some adaptations are necessary. In this way, sown season and background normal effects were inserted in model 1, where flat prior was assumed for sown variance and $p\left(\sigma_{b}^{2}\right) \propto 1 / \sigma_{c}^{2}$ for background variance.

For the analysis, a program was developed using the SAS/IML (SAS Institute, 2000) package. Chains of different sizes were used according to the analysis (per cross or combined). The chain sizes as well as the burn-in and jump process were obtained as suggested by Raftery and Lewin (1992). For the stationarity analysis of the chains we used the criterion suggested by Gelman and Rubin (1992) and Brooks and Gelman (1998) using the Bayesian Output Analysis package (BOA) available for platform R.

\section{RESULTS}

The chain sizes as well as the "burn-in" and "jump" process varied according to the cross and sown season (Table 1). It was observed that the largest chain sizes were required in second season for convergence. The stationarity of the chains was reached earliest for the first season and cross GNS31 x GNS30. On the other hand, for join analysis a chain of 510,000 samples was required with burn-in of 10,000 and storage of one sample in 50 sampled.

Disease incidence in the two backgrounds (GNS31 x GNS84 and GNS31 x GNS30) increased in the second sowing, with a mean of 4.81 and 5.75 for first and second sowing, respec- 
tively. This found was probably due to the greater inoculum pressure in the experiments conducted at second sowing. The susceptibility of the populations that arose from GNS31 x GNS30 cross was slightly higher than ones coming from GNS31 x GNS84 cross (Figure 1). This finding suggests that the GNS84 line could be more resistant than the GNS30. The mean and median of the background variance were 3.871 and 0.428 . This difference was due to shape of the sampled inverted chi-square distribution; in other words, this divergence could be caused by a restricted number of degrees of freedom (d.f. $=2$ ) contained in background effects (two backgrounds), resulting in an L-shaped inverted chi-square. Under this scenario, the median might present a superior measure of position of an L-shaped distribution than the mean measure.

\begin{tabular}{|c|c|c|c|}
\hline & GNS31 x GNS84 & & \\
\hline Grown & & Chain & MPRSF \\
\hline Season 1 & & 330000 & 1.000 \\
\hline \multirow[t]{2}{*}{ Season 2} & & 400000 & 1.000 \\
\hline & GNS31 x GNS30 & & \\
\hline Grown & & Chain & MPRSF \\
\hline Season 1 & & 95000 & 1.000 \\
\hline \multirow[t]{3}{*}{ Season 2} & & 160000 & 1.000 \\
\hline & Join & & \\
\hline & & Chain & MPRSF \\
\hline Season $1+$ Season 2 & & 510000 & 1.000 \\
\hline
\end{tabular}

$\mathrm{MPSRF}=$ multivariate potential scale reduction factor.

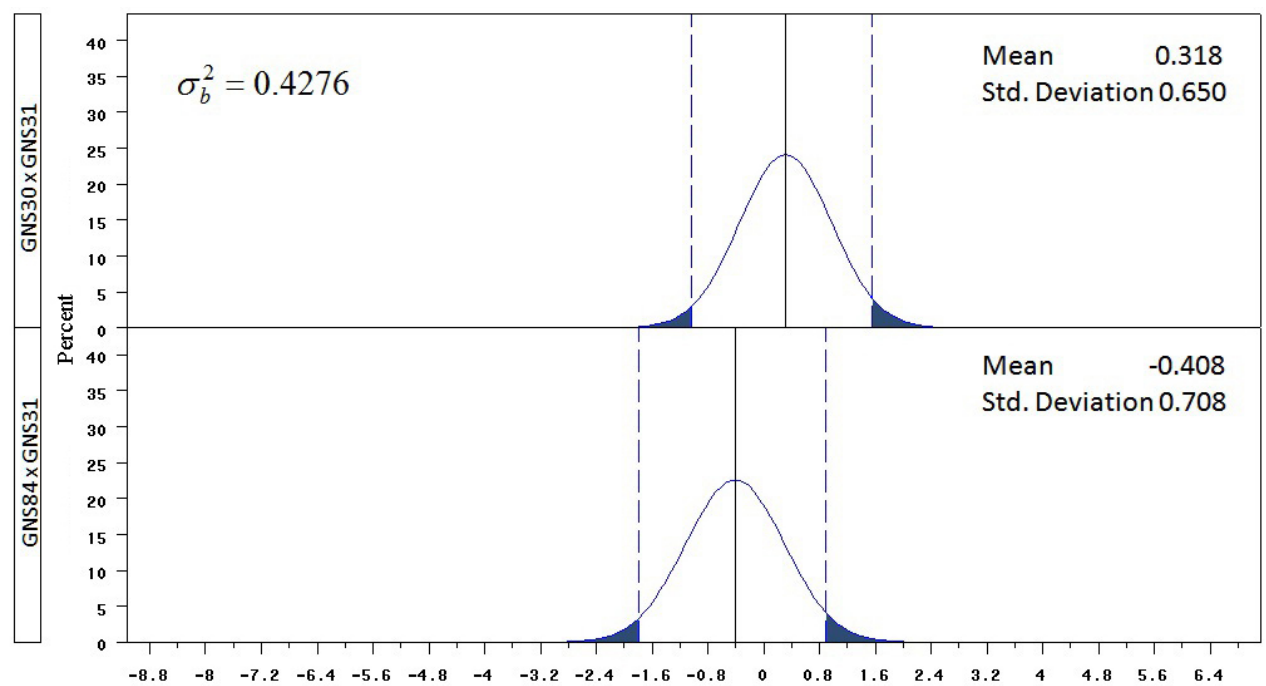

Figure 1. Background effects correspondent to GNS84 x GNS31 and GNS30 x GNS31 crosses and background variance.

Independent of the season assessed, high disease severity was observed in the susceptible line GNS31 - common to both crosses - ranging from 8.85 to 8.95 . The disease severity related to the resistant line GNS30 was of 1.11. Also, the line GNS84 had a severity note of 
1.10 and the performance of the $\mathrm{F}_{1}$ hybrid was intermediate between mid-parent and resistant line, presenting a severity of 3.20 and 2.15 in the first and second season, respectively.

The results based on $F_{1}$ and $F_{2}$ means suggest, at first instance, the presence of non-additive effects on genetic resistance for C. zeae-maydis, i.e., imply partial dominance since only for the GNS31 x GNS30 cross - in the first season - the mean of the $\mathrm{F}_{1}$ generation was similar to the mean of the $\mathrm{F}_{2}$ generation and mid-parent as well. However, the individual and join analysis revealed a strong influence of the additive effect on the genetic control of GLS.

It was observed that the magnitude of additive effects was mostly positive and different from zero (Table 2 and Figure 2). Moreover, the additive effect observed in the join analysis was similar to that obtained in the grown 1 . The results of individual and join analysis suggest that additive effect has significant influences on GLS resistance. The additive variance ranged from 0.86 to 34.8 evidencing the importance of this effect on genetic control of GLS. The additive heritability was highlighting high since it presented $95 \%$ of its values higher than 0.23 converging in a beta posterior probability distribution with parameters $\alpha=1.36$ and $\beta=$ 0.42 (Figure 3).

\begin{tabular}{|c|c|c|c|c|c|c|}
\hline \multirow[t]{4}{*}{ Effects/parameters } & \multicolumn{6}{|c|}{ GNS31 x GNS84 } \\
\hline & \multicolumn{3}{|c|}{ Sown 1} & \multicolumn{3}{|c|}{ Sown 2} \\
\hline & \multirow[t]{2}{*}{ Mean } & \multicolumn{2}{|c|}{ Quantiles } & \multirow[t]{2}{*}{ Mean } & \multicolumn{2}{|c|}{ Quantiles } \\
\hline & & 0.05 & 0.95 & & 0.05 & 0.95 \\
\hline$a$ & 3.755 & 2.293 & 4.656 & 2.302 & -0.216 & 5.962 \\
\hline$d$ & -0.365 & -2.050 & 0.104 & -0.450 & -5.540 & 2.696 \\
\hline$a a$ & 0.083 & -0.178 & 0.909 & -0.441 & -4.599 & 1.668 \\
\hline$a d$ & 0.242 & -0.350 & 2.242 & 1.817 & -3.364 & 14.131 \\
\hline$d d$ & -0.430 & -1.811 & 0.078 & -0.761 & -6.012 & 1.703 \\
\hline & Mean & & Median & Mean & & Median \\
\hline$\sigma_{\mathrm{a}}^{2}$ & $4.1 \times 10^{4}$ & & 30.100 & 49.104 & & 7.300 \\
\hline$\sigma_{\mathrm{d}}^{2}$ & $3.9 \times 10^{5}$ & & $1.3 \times 10^{-4}$ & 30.104 & & 0.150 \\
\hline$\sigma_{\mathrm{aa}}^{2}$ & $3.4 \times 10^{3}$ & & $6.9 \times 10^{-6}$ & 56.103 & & 0.088 \\
\hline$\sigma_{\mathrm{ad}}^{2}$ & $3.5 \times 10^{3}$ & & $1.2 \times 10^{-5}$ & 62.104 & & 0.440 \\
\hline$\sigma_{\mathrm{dd}}^{2}$ & $1.3 \times 10^{3}$ & & $2.4 \times 10^{-4}$ & 77.104 & & 0.180 \\
\hline \multirow{5}{*}{$\begin{array}{l}\sigma_{\text {dd }} \\
\sigma^{2} \\
\end{array}$} & 1.897 & & 0.579 & 19.458 & & 10.996 \\
\hline & \multicolumn{6}{|c|}{ GNS31 x GNS30 } \\
\hline & \multicolumn{3}{|c|}{ Sown 1} & \multicolumn{3}{|c|}{ Sown 2} \\
\hline & \multirow[t]{2}{*}{ Mean } & \multicolumn{2}{|c|}{ Quantiles } & \multirow[t]{2}{*}{ Mean } & \multicolumn{2}{|c|}{ Quantiles } \\
\hline & & 0.05 & 0.95 & & 0.05 & 0.95 \\
\hline $\bar{a}$ & 3.947 & 3.360 & 4.608 & 1.943 & -0.021 & 4.801 \\
\hline$d$ & 0.144 & -0.208 & 0.975 & 0.325 & -0.362 & 2.934 \\
\hline$a a$ & 0.257 & -0.056 & 1.280 & -0.464 & -3.337 & 0.214 \\
\hline$a d$ & 0.926 & -0.091 & 4.781 & -0.455 & -5.559 & 1.213 \\
\hline \multirow[t]{2}{*}{$d d$} & 0.046 & -0.127 & 0.236 & -0.008 & -1.000 & 1.376 \\
\hline & Mean & & Median & Mean & & Median \\
\hline$\sigma_{a}^{2}$ & $5.9 \times 10^{5}$ & & 34.600 & $4.5 \times 10^{4}$ & & 4.41 \\
\hline$\sigma_{\mathrm{d}}^{2}$ & $8.2 \times 10^{2}$ & & $2.1 \times 10^{-5}$ & $1.5 \times 10^{3}$ & & $1.31 \times 10^{-5}$ \\
\hline$\sigma_{\text {aa }}^{2}$ & $1.3 \times 10^{3}$ & & $5.8 \times 10^{-5}$ & $1.2 \times 10^{3}$ & & $3.5 \times 10^{-5}$ \\
\hline$\sigma_{\text {ad }}^{2}$ & $5.6 \times 10^{3}$ & & 0.006 & $9.1 \times 10^{3}$ & & $3.3 \times 10^{-5}$ \\
\hline$\sigma_{\mathrm{dd}}^{2}$ & $1.1 \times 10^{3}$ & & $2.7 \times 10^{-5}$ & $9.8 \times 10^{3}$ & & $1.1 \times 10^{-5}$ \\
\hline$\sigma^{2}$ & 0.609 & & 0.230 & 12.880 & & 6.966 \\
\hline
\end{tabular}




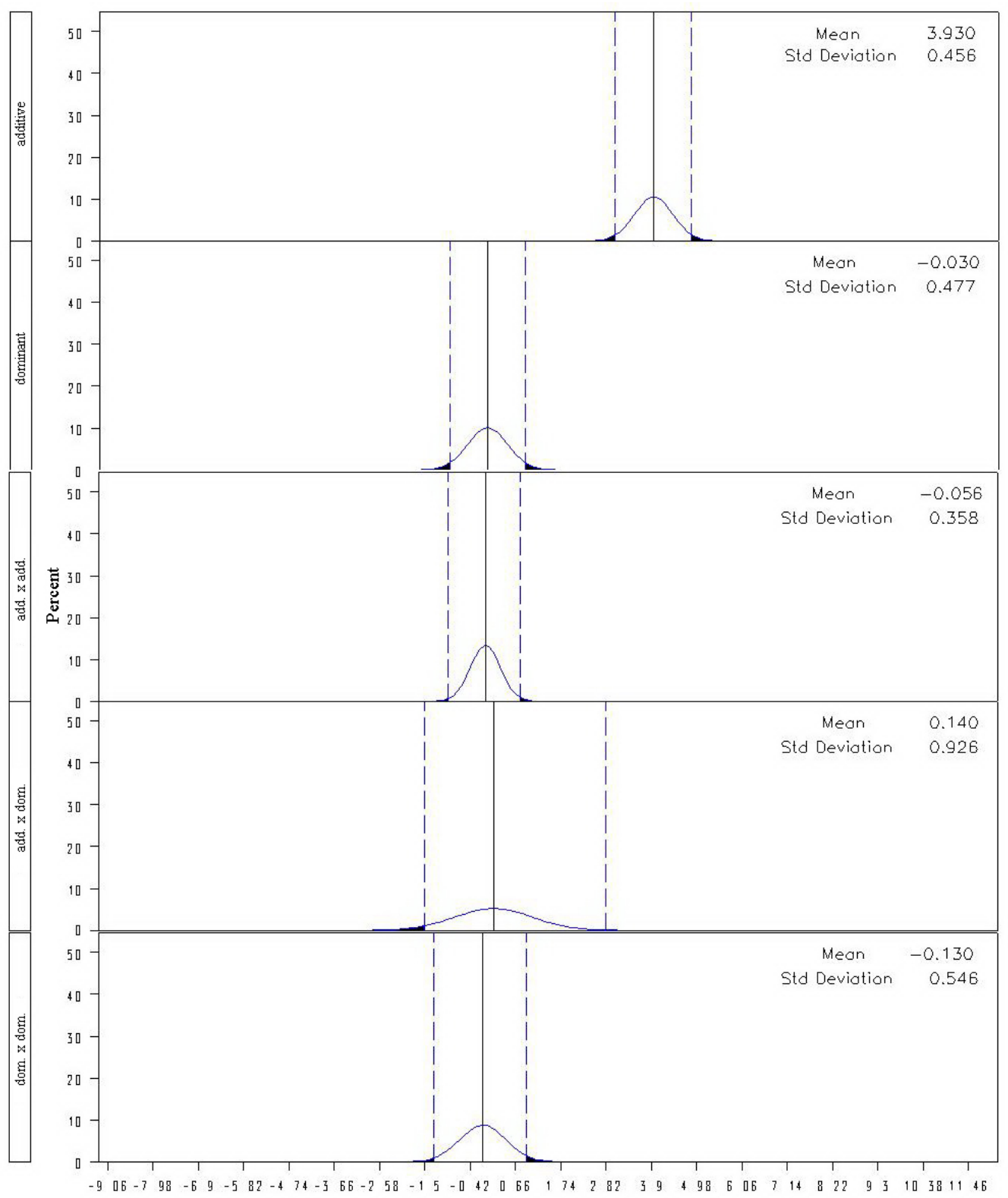

Figure 2. Posterior probability distributions of additive, dominant and epistatic effects and their variances obtained in join analysis. 


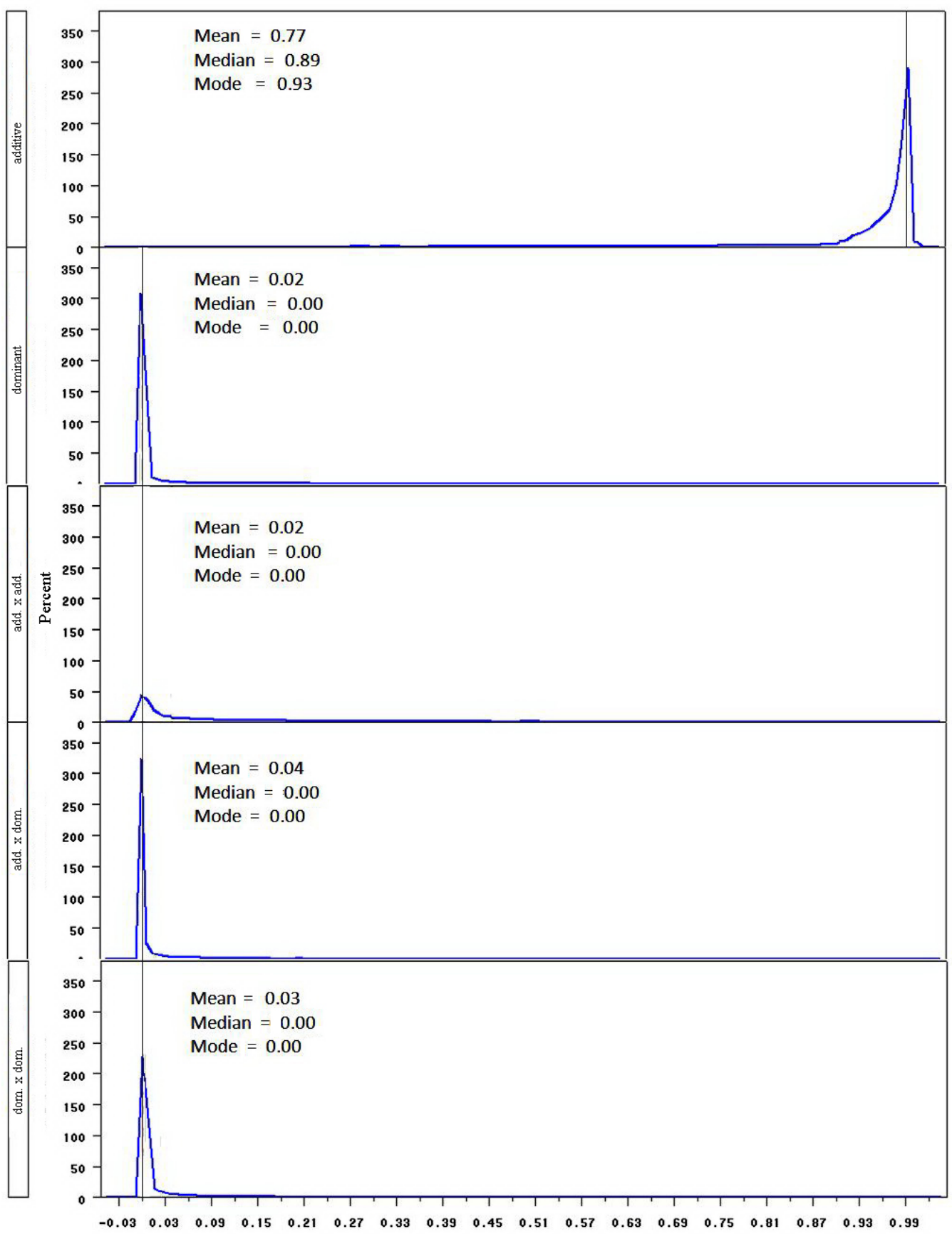

Figure 3. Posterior probability distributions of additive, dominant and epistatic heritability obtained in join analysis. 
In terms of magnitude, the dominance effect presented a low influence on GLS resistance and was variable in direction according to background analyzed; being negative for GNS84 x GNS31 and positive for GNS30 x GNS31. The dominant variance was nearest of zero in individuals and join analysis, ranging from $2.61 \times 10^{-6}$ to 0.002 (Table 2 and Figure 2). Dominance shrinkage effect occurred due to magnitude of its variance that was lowest in relation to residual variance. It is an advantage of Bayesian shrinkage analysis since effects of marginal importance are more penalized by residual variance than those with significant importance. In addition to this, in the joint analysis, the dominance effects obtained for both background 1 and 2 were cancelled out since it presented opposite sign - presented negative effect in the cross GNS84 x GNS31 and positive effect in GNS84 x GNS31 cross (Table 2). Likewise, the dominance heritability was extremely low with median and mode equal to zero reflecting a beta posterior probability distribution with parameters $\alpha=0.06$ and $\beta=2.22$.

Therefore, it can be inferred that dominance effects exhibit a minor effect in the control of GLS resistance.

In the second grown season, the model adjustment was less effective reflecting in a high residual variance. Not by chance, in this circumstance, more influence of the epistatic effects was observed in detriment of additive effect: the additive $\mathrm{x}$ dominance epistasis was equivalent to additive effect in GNS84 x GNS31 background. Notwithstanding the additive x dominance effect had not been significantly different from zero by its posterior probability distribution, it was mostly larger than dominance, additive $\mathrm{x}$ additive and dominance $\mathrm{x}$ dominance ones. These results may suggest that under high inoculum pressure, the host might make use of complex genetics interaction in order to increase the resistance to GLS. However, in general, additive $\mathrm{x}$ dominance had minor influence on GLS genetic control, presenting null heritability (Figure 3).

The additional epistatic effects, such as additive $\mathrm{x}$ additive and dominance $\mathrm{x}$ dominance, demonstrated marginal importance in the genetic control of the GLS. These effects were shrunken close to zero converging in variances close to zero as well (Figure 2). In addition, the heritability posterior distribution, considering all epistatic effects, converged in an L-shaped beta posterior distribution with parameters $\alpha<0.2$ and $\beta \geq 1.1$ (Figure 3).

Thus, the results obtained in this study suggest that genetic control of GLS resistance is strongly influenced by additive genes and just only in extraordinary circumstance by additive $\mathrm{x}$ dominance epistatic effect.

\section{DISCUSSION}

The use of Bayesian inference to study trait inheritance has been extremely useful in animal (Kadarmideen and Janss, 2005), and plant breeding (Silva et al., 2009). Among the already known advantages over frequentist analysis, the potential use of priors, flexibility in complex models, accurate credibility intervals, smaller square error estimators, and others can be highlighted. Another advantage of Bayesian shrinkage analysis is the direct estimate of additive, dominant, and epistatic variance in a single-model, which is unfeasible in common least square. In addition, direct inferences on posterior probability distribution and accurate credibility intervals make this analysis very attractive in terms of inference on genetic parameters. Also, it is possible updating the breeder's knowledge about genetic control of GLS resistance; based on our results, informative priors might be built for main and epistatic effects in further studies. 
The join-scaling test suggested by Mather and Jinks (1971) is commonly used for parameter estimates in study of genetic control; however, this approach presents several disadvantages that might restrict its application. This methodology cannot directly handle linear models where the number of parameters is larger/equal than the number of observations. In this circumstance, a statistical test might be restrictive due to its restricted number of degrees of freedom and the full-model intractable for test. In addition, the epistatic effects are assumed as a non-adjustment of the additive-dominant model, forcing the full-model explains $100 \%$ of phenotypic variance. To get around this limitation, model selection is normally applied; however, it does not effectively solve the full-model test problem in over-parametric models.

Bayesian shrinkage analysis is an alternative to model selection since it is a freemodel selection methodology. In this approach, epistatic parameters and their variances can be adjusted in a single-over-parameterized model where whole probability inferences are built given the phenotypic observations and uncertain about additive, dominant and epistatic effects. Due to these several advantages Bayesian shrinkage analysis was applied in this study.

Even with non-informative prior distributions used for all parameters, the results of the Bayesian shrinkage analysis were very different to the ones expected by the weighted-least squares method. For example, in Bayesian shrinkage analysis each effect is directly penalized by its heritability, if so, those parameters with marginal effects have low variance, low heritability and hence are shrunken to nearest zero values. Herein, for instance, dominant and epistatic effects were shrunken for values near zero presenting roughly heritability equal to zero. On the other hand, in complete-scaling test obtained by weighted-least square, these effects were extremely high achieving 2 to 3 times the additive magnitude even when the adjustment of additive-dominant model explained about $95 \%$ of the phenotypic variance (data not shown). Nevertheless, it is probable that when the complete-model was adjusted in joinscaling test, additive, dominant and epistatic effects were over- or underestimated since these ones are obtained without error in least square model, i.e., the contrasts of the parameters explained $100 \%$ of phenotypic variance.

However, the divergences between weighted-least square and Bayesian results were not absolute. The additive effect obtained in Bayesian shrinkage analysis, join-scaling test and additive/dominant scaling test was very similar, i.e., in a scaling test it ranged from 3.86 to 3.93 , varying according to the grown season and background. These values are inside of Bayesian additive credibility interval (95\%) shown in Figure 3. The further parameters obtained from weighted-least square, on the other hand, were outside of Bayesian credibility interval, and if so, it is possible to infer that scaling estimates for dominant and epistatic effects obtained herein by join-scaling test are improbable given the actual data set.

Predominance of additive effects in the genetic control of GLS resistance was observed in this study, as well as a minor influence of dominant and epistatic effects. Taking these last components into account, a moderate influence of the additive $\mathrm{x}$ dominant epistatic effect was observed on high inoculum pressure. This kind of epistatic effect influencing GLS resistance is not uncommon in the literature, i.e., Coates and White (1998) found influence of dominant $\mathrm{x}$ additive epistasis on GLS resistance as well.

Previous studies of QTL mapping for GLS resistance largely corroborate the results observed herein. In some of these reports, additive gene effects were linked with QTL regions mapped on chromosome 2 by Bubeck et al. (1993), on chromosome 1 by Saghai-Maroof et al. (1996) and also on chromosome 4 by Gordon et al. (2004). Our results are consistent with 
most of the reports in the literature, in that previous studies revealed that additive effects account for more than $90 \%$ of the variability among generations and that GLS resistant could be: i) inherited additively, ii) controlled by additive gene action and presenting minor dominant and epistatic contributions, iii) associated with the general combining ability of inbred lines, and iv) controlled by few effective factors (Clement et al., 2000; Julliati et al., 2009; Pozar et al., 2009). The results and inferences about GLS genetic resistance obtained in this study would be different from previously reported studies if least square estimates were applied, since a high influence of epistasis was observed on scaling-test (data not shown). Our results probably would be similar to those obtained by Coates and White (1998). These authors, applying join-scaling test by weighted-least square (full-model explaining $100 \%$ of phenotypic variance) in various backgrounds, observed a non-negligible epistasis in GLS genetic control. However, as previously highlighted, significant epistasis has not been observed in QTL mapping studies for GLS resistance.

Given the additive action of the genes involved in GLS and high additive heritability observed in this study, mass selection or another recurrent selection method could be indicated as breeding strategy to obtain resistant breeding populations for use per se or to obtain resistant inbred lines to be used in single-cross hybrids. However, since epistatic effects can emerge under high inoculum pressure, these effects cannot be ignored completely.

Based on these results, one can infer that Bayesian shrinkage analysis is an excellent approach to handle with complex models in study of genetic control in GLS. Gray leaf spot resistance was predominantly additive, whereas the influences of both dominant and epistatic effects on the genetic control of this disease were insignificant.

\section{ACKNOWLEDGMENTS}

The authors acknowledge the support of the Brazilian Federal Agency for Support and Evaluation of Graduate Education - CAPES and FAPEMIG (Research Support Foundation of Minas Gerais).

\section{REFERENCES}

Brito AH, Von Pinho RG, Souza Filho AX and Altoé TF (2008). Avaliação da severidade da Cercosporiose e rendimento de grãos em híbridos comerciais de milho. Rev. Bras. Milho Sorgo 7: 19-31.

Brooks SP and Gelman A (1998). General methods for monitoring convergence of iterative simulations. J. Comput. Graph. Stat. 7: 434-455.

Bubeck DM, Goodman MM, Beavis WD and Grant D (1993). Quantitative trait loci controlling resistance to gray leaf spot in maize. Crop Sci. 33: 838-847.

Clements MJ, Dudley JW and White DG (2000). Quantitative trait loci associated with resistance to gray leaf spot of corn. Phytopathology 90: 1018-1025.

Coates ST and White DG (1998). Inheritance of resistance to gray leaf spot in crosses involving selected resistant inbred lines of corn. Phytopathology 88: 972-982.

Derera J, Tongoona P, Kevin VP, Vivek B, et al. (2008). Gene action controlling gray leaf spot resistance in Southern African maize germplasm. Crop Sci. 48: 93-98.

Gelman A and Rubin DB (1992). Inference from iterative simulation using multiple sequences. Stat. Sci. 7: 457-472.

Gordon GS, Bartsch M, Matties I, Gevers HO, et al. (2004). Linkage of molecular markers to Cercospora zeae-maydis resistance in maize. Crop Sci. 44: 628-636.

Janss LL, Van Arendonk JA and Brascamp EW (1997). Bayesian statistical analyses for presence of single genes affecting meat quality traits in a crossed pig population. Genetics 145 : 395-408.

Juliatti FC, Appelt CCNS, Brito CH, Gomes LS, et al. (2004). Controle da feosféria, ferrugem comum e cercosporiose pelo 
uso da resistência genética, fungicidas e épocas de aplicação na cultura do milho. Biosci. J. 20: 45-54.

Juliatti FC, Pedrosa MG, Silva HD and Silva JVC (2009). Genetic mapping for resistance to gray leaf spot in maize. Euphytica 169: 227-238.

Kadarmideen HN and Janss LL (2005). Evidence of a major gene from Bayesian segregation analyses of liability to osteochondral diseases in pigs. Genetics 171: 1195-1206.

Mather K and Jinks JL (1971). Biometrical Genetics. Chapman and Hall, London.

Menkir A and Ayodele M (2005). Genetic analysis of resistance to gray leaf spot of midaltitude maize inbred lines. Crop Sci. 45: 163-170.

Pozar G, Butruille D, Silva HD, McCuddin ZP, et al. (2009). Mapping and validation of quantitative trait loci for resistance to Cercospora zeae-maydis infection in tropical maize (Zea mays L.). Theor. Appl. Genet. 118: 553-564.

Raftery AE and Lewis SM (1992). How Many Iterations in the Gibbs Sampler? In: Bayesian Statistics 4 (Bernardo JM, Berger JO, Dawid AP and Smith AFM, eds.). Oxford University Press, Oxford, 763-773.

Saghai-Maroof MA, Yue YG, Xiang ZX, Stromberg EL, et al. (1996). Identification of quantitative trait loci controlling resistance to gray leaf spot disease in maize. Theor. Appl. Genet. 93: 539-546.

SAS Institute (2000). Version 8. SAS Institute Inc., Cary.

Silva MIS, Bearzoti E and Bueno-Filho JSS (2009). Análise bayesiana do modelo de herança monogênica no melhoramento vegetal: um exemplo com abobrinha. Cienc. Agrotecnol. 33: 1463-1468.

Von Pinho RG, Ramalho MAP, Resende IC, Silva HP, et al. (2001). Reação de híbridos comerciais de milho às ferrugens polissora e tropical. Pesq. Agropec. Bras. 36: 439-445.

Xu S (2003). Estimating polygenic effects using markers of the entire genome. Genetics 163: 789-801. 